\title{
Design of High data Rate FM-QCSK Chaotic Communication System
}

\author{
Hikmat N. Abdullah \\ Department of Electrical En gineering, Al-Mustansiry ah University, Baghdad, P. O. Box 14150, Iraq
}

\begin{abstract}
The frequency modulated quadrature chaos shift keying FM-QCSK system is one of the most efficient systems in chaotic literature. One of the problems in this system is that half the bit duration is used for sending a chaotic reference signal which leads to increase the energy losses and reduces the data rate. In this paper, a novel scheme to enhance the performance of FM-QCSK system has been proposed. With the proposed scheme, FM-QCSK would be able to operate at higher data rates with reduced bit-error probability BER and energy consumption. The basic modification introduced by the proposed scheme is the use one reference chaotic chip to transmit multi information-bearing chips in both in-phase and quadrature-phase channels. The results showed that the proposed scheme have achieved more than $3 \mathrm{~dB}$ and $5 \mathrm{~dB}$ gains in SNR for AWGN and Rayleigh multipath fading channels respectively at BER $=10^{-3}$ over the conventional one. The results also showed that the optimum number in formation-bearing chip can be send per reference is 8 . The theoretical expression of BER in AW GN channel has also been derived for the proposed scheme.
\end{abstract}

Keywords Chaotic Communication, Quadrature Chaos Shift Key ing, Orthogonal

\section{Introduction}

With researching in the chaos-based communication systems, more and more methods are applied in this area and more and more modulation schemes are proposed. In which differential chaotic shift keying (DCSK)[1], using correlation to demodulate, was proposed to solve the problem of chaotic synchronization. These commun ication systems have the wide band characteristic of chaotic signal and advance in resisting multipath fading[2]. To enhance the noise performance of DCSK, FM-DCSK[3] scheme was proposed where frequency modulation is utilized to achieve constant energy per bit for the chaotic carrier. DCSK is a transmitted-reference signaling scheme. For each symbol period, the DCSK signal consists of a piece of chaotic waveform (called reference chip), followed by its non-inverted or inverted copy (called information bearing chip), depending on the binary symbol ("0" or " 1 ") to be transmitted.

QCSK[4] (Quadrature Chaos Shift Keying) which can transmit 2 bits in a sample function was designed by Zbigniew Galais and Gian Maggio to improve the speed of chaos shift keying in 2001. Then in 2006, Yiwei Zhang devised FM-QCSK[5], which enhanced the noise performance of QCSK by using frequency modulation, because it

* Corresponding author:

hikmat_04@yahoo.com (Hikmat N. Abdullah)

Published online at http://journal.sapub.org/jwnc

Copyright (C) 2012 Scientific \& Academic Publishing. All Rights Reserved generates constant energy per bit and its frequency spectrum is wideband.

Several different methods have been proposed in the literature to increase the data rate in both DCSK and QCSK systems[6-10]. The simplest options consist of scaling the information and/or the reference parts of the signal like the work in[6-8]. More sophisticated approaches use multi-level signal constellations like QAM, M-ary phase shift keying or multi-chaotic basis functions by dividing the symbol period into M-ary time slots[9] or by defining a set of orthogonal vectors[10]. All the previous works constrained; in principle, on increasing the space dimension of the chaotic carrier as a way to increase the data rate. However, there were no real concerns to the number of information-bearing chips which are referred to one reference. In this paper, an enhanced version of FM-QCSK scheme is proposed by using the idea of transmitting more than one information-bearing chip for one reference chip and averaging the correlation results for both the in-phase and quadrature phase channels to improve the noise performance, reduce the energy consumption and to increase the data rate of FM-QCSK.

\section{Background Theory}

Z. Galias, and G. M. Maggio described in their article[4] that the orthogonal basis of chaotic functions $[\mathrm{x}(\mathrm{t})$ and $\mathrm{y}(\mathrm{t})]$ can be easily generated through Hilbert transform and this chaotic sample functions over the interval $[0, T]$ can be defined as follows: 


$$
C \equiv\left[c_{x}(t), c_{y}(t)\right]=\left[\frac{1}{E_{b}} x(t), \frac{1}{E_{b}} y(t)\right]
$$

$E_{b}$ is the energy associated with chaotic signal $x(t)$ and $y(t)$ in a sample period $[0, T]$. Therefore, the formula can be defined as:

$$
\int_{0}^{T} c_{x}^{2}(t) d t=\int_{0}^{T} c_{y}^{2}(t) d t=1
$$

The quadrature signal of $x(t)$ is $y(t)$, so we can get the following equation:

$$
\int_{0}^{T} c_{x}(t) c_{y}(t) d t=0
$$

The corresponding to two typical versions of QCSK modulation, constellations distribution is shown in Figure 1, where dashed lines represent the decision boundaries respectively.

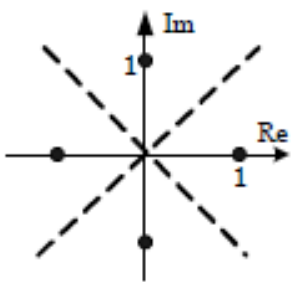

(a) QCSK

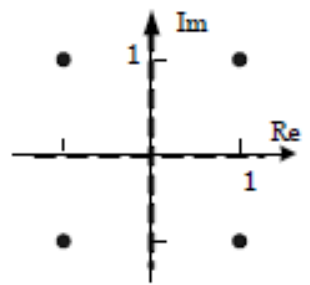

(b) $\mathrm{QCSK}^{*}$
Figure 1. Two versions of the QCSK constellations

In a FM-QCSK system, the chaotic reference chip $c_{x}(t)$ which is modulated by FM modulator is transmitted in the first half symbol period while the in formation chip $\mathrm{m}_{\mathrm{i}}(\mathrm{t})$ which is also modulated by FM is transmitted in the second half. The ith symbol of the modulated information signal $\mathrm{m}_{\mathrm{i}}(\mathrm{t})$ can be defined as follows:

$$
m_{i}(t)=a_{i}(t)+b_{i}(t)
$$

Therefore, signals transmitted by FM-QCSK can be expressed in a symbol period T:

$$
\begin{aligned}
& S_{F M-Q C S K}(t)= \\
& \begin{cases}\sqrt{E_{b}} c_{x}(t) & 0 \leq t<\frac{T}{2} \\
\sqrt{E_{b}}\left[a_{i} c_{x}\left(t-\frac{T}{2}\right)+b_{i} c_{y}\left(t-\frac{T}{2}\right)\right] & \frac{T}{2} \leq t<T\end{cases}
\end{aligned}
$$

In the above formula, $\mathrm{a}_{\mathrm{i}}$ and $\mathrm{b}_{\mathrm{i}}$ are the mapping coordinate of signal symbol in constellation. The combinations of $a_{i}$ and $b_{i}$ that denote the different symbols are shown in Table 1.

Table 1. Corresponding $a_{i}$ and $b_{i}$ for each quaternary symbol

\begin{tabular}{|c|cccc|}
\hline Symbol & 00 & 01 & 10 & 11 \\
\hline QCSK & $1+0 i$ & $0+1 i$ & $0-1 i$ & $-1+0 i$ \\
\hline QCSK $^{*}$ & $(+1+i) / \sqrt{2}$ & $(-1+i) / \sqrt{2}$ & $(+1-i) / \sqrt{2}$ & $(-1-i) / \sqrt{2}$ \\
\hline
\end{tabular}

\section{Enhanced FM-QCSK Modulation}

The time slots of the original FM-QCSK signal are shown in the upper part of Figure 2. In this figure, $R_{i}$ denote the reference chips of the ith bit while $a_{i}$ and $b_{i}$ denote the information-bearing chips of the ith bit for the in-phase and quadrature-phase respectively. One of the drawbacks of conventional FM-QCSK is that every information bit is transmitted by two chips (the reference and information-bearing chips). Hence, the bit rate (as well as the symbol rate) is halved and the transmitted energy per bit is doubled compared to the conventional binary modulation schemes where every sample function represents one bit. A possible enhancement of the conventional FM-QCSK scheme is as follows: instead of transmitting only one information-bearing chip after one reference chip, $\mathrm{N}$ bits in each

\begin{tabular}{|c|c|c|c|c|c|c|}
\hline $\mathrm{R}_{1}$ & $a_{1}$ & $\mathbf{R}_{2}$ & $\mathrm{a}_{2}$ & & $\mathbf{R}_{\mathbf{M}}$ & I - channel \\
\hline & $b_{1}$ & & $b_{2}$ & & $b_{M}$ & Q - channel \\
\hline & QCSK & & & & & \\
\hline$\stackrel{\mathrm{T}_{\mathrm{s}}}{\longleftrightarrow}$ & & & & & & \\
\hline $\mathbf{R}_{1}$ & $a_{1,1}$ & $a_{1,2}$ & $a_{1,3}$ & & $a_{1, N}$ & I - channel \\
\hline & $\mathrm{b}_{1,1}$ & $b_{1,2}$ & $b_{1,3}$ & & $b_{1, N}$ & Q - channel \\
\hline
\end{tabular}
channel are transmitted using the same reference. Th is idea is first discussed in[6], but tested in DCSK system where there are no orthogonal chaotic carriers and symbol transmission is introduced as in FM-QCSK.$$
\mathrm{E}_{\mathrm{s}}
$$

Figure 2. Typical time slots of the conventional FM-QCSK and the enhanced FM-QCSK systems

The waveform of the enhanced FM-QCSK scheme is shown in the lower part of Figure 2, where $T_{s}$ denote the duration of one chip and $E_{s}$ is the energy carried by chip. Observe that in a block containing $\mathrm{N}+1$ chips for every chip, except the first one, carries information. The enhanced modulation scheme offers two advantages over the conventional one: firstly, the bit duration $\mathrm{T}$ is decreased fro $\mathrm{m}_{2} \mathrm{~T}_{\mathrm{s}}$ to $((\mathrm{N}+1) / \mathrm{N}) \mathrm{T}_{\mathrm{s}}$, i.e., the data rate is increased. Secondly, the transmitted energy per bit $\mathrm{E}_{\mathrm{b}}$ is reduced from $2 \mathrm{E}_{\mathrm{s}}$ to $((\mathrm{N}+1) / \mathrm{N}) \mathrm{E}_{\mathrm{s}}$ (or equivalently energy per symbol is reduced from $4 \mathrm{Es}$ to $2((\mathrm{~N}+1) / \mathrm{N}) \mathrm{E}_{\mathrm{s}}$. However, the enhanced system may also suffers from the drawbacks of increasing periodic component at frequency $1 / T_{\mathrm{s}}$ and its harmonics as well as the increase in the system complexity.

Let $\mathrm{s}_{\mathrm{m}}(\mathrm{t})$ denote the transmitted signal in the mth time slot $t \in\left[m T_{s},(m+1) T_{s}\right]$ for the in-phase channel. Hence $\mathrm{s}_{0}(\mathrm{t})=\mathrm{c}_{\mathrm{x}}(\mathrm{t})$ is the reference chip and $\mathrm{s}_{\mathrm{m}}(\mathrm{t})={ }_{\mathrm{s}} \mathrm{s}_{0}\left(\mathrm{t}-\mathrm{mT}_{\mathrm{s}}\right)$ is the information bearing chip for bit $\mathrm{D}_{\mathrm{m}}$. Let $\check{\mathrm{r}}_{\mathrm{m}}(\mathrm{t}), \mathrm{m}=0,1, \ldots, \mathrm{N}$, denote the signal received in the mth time slot. The correlation between the kth and lth chips be denoted by: 


$$
z_{k, l_{l}}=\breve{r}_{k}(t) * \breve{r}_{l}(t) \int_{0}^{\frac{T}{N+1}} \breve{r}_{k}\left(t-\frac{k}{N+1} T\right) \breve{r}_{l}\left(t-\frac{l}{N+1} T\right) d t
$$

There are two observation signals of the mth symbol at the receiver for the in-phase and quadrature-phase channels. These are defined as:

$$
\begin{aligned}
z_{0, m_{I}} & =\breve{r}_{0}(t) * \breve{r}_{m_{I}}(t) \\
z_{0, m_{Q}} & =\breve{r}_{0}(t) * \breve{r}_{m_{Q}}(t)
\end{aligned}
$$

Note that $\breve{\mathrm{r}}_{\mathrm{m}}(\mathrm{t})$ and $\check{\mathrm{r}}_{\mathrm{mI}}(\mathrm{t})$ signals are the same while $\check{\mathrm{r}}_{\mathrm{mQ}}(\mathrm{t})$ signal is $\check{\mathrm{r}}_{\mathrm{m}}(\mathrm{t})$ signal after applying Hilbert trans form.

\section{Transmitter and Receiver Configurations}

Figure 3 shows the implementation of the enhanced FM-QCSK modulator. First the chaos signal is applied to an FM modulator to get constant energy per bit. The enhanced modulator contains of a delay with $\mathrm{N}$ taps, the output of each tap is input to a QCSK modulator. The trans mission of each $\mathrm{N}$ symbols is preceded by a reference ch ip s $0(\mathrm{t})$, after which the information-bearing chips $s m(t)$ are transmitted. This is done by changing the switch positions at each Ts time instants.

The block diagram of the demodulator contains $\mathrm{N}$ delay lines and correlator pairs as shown in Figure 4. The correlator pair outputs sampled at $\mathrm{kTs}, \mathrm{k}=1,2, \ldots \mathrm{N}$ constitute the elements of observation vectors for in-pahse and quadrature phase channels. They are denoted by $\mathrm{z}_{1 \mathrm{I}}, \mathrm{z}_{1 \mathrm{Q}}, \mathrm{z}_{2 \mathrm{I}}, \mathrm{z}_{2 \mathrm{Q}} \ldots . . \mathrm{Z}_{\mathrm{NI}}$, $\mathrm{Z}_{\mathrm{NQ}}$ in Figure 3 and their values are given in Table 2. The transmitted information is carried by the sign and relative orthogonality of the correlation between the reference and information-bearing chips. This information is available for the mth symbol at the output of the mth correlator pair at $(\mathrm{m}+1)$ th sampling time instant as shown in Table 2. The estimated information is denoted by a vector $\check{\mathrm{D}}=\left(\check{\mathrm{D}}_{1}, \check{\mathrm{D}}_{2} \ldots \ldots \check{\mathrm{D}}_{\mathrm{N}}\right)$ and obtained from the output of symbol/bit converter.

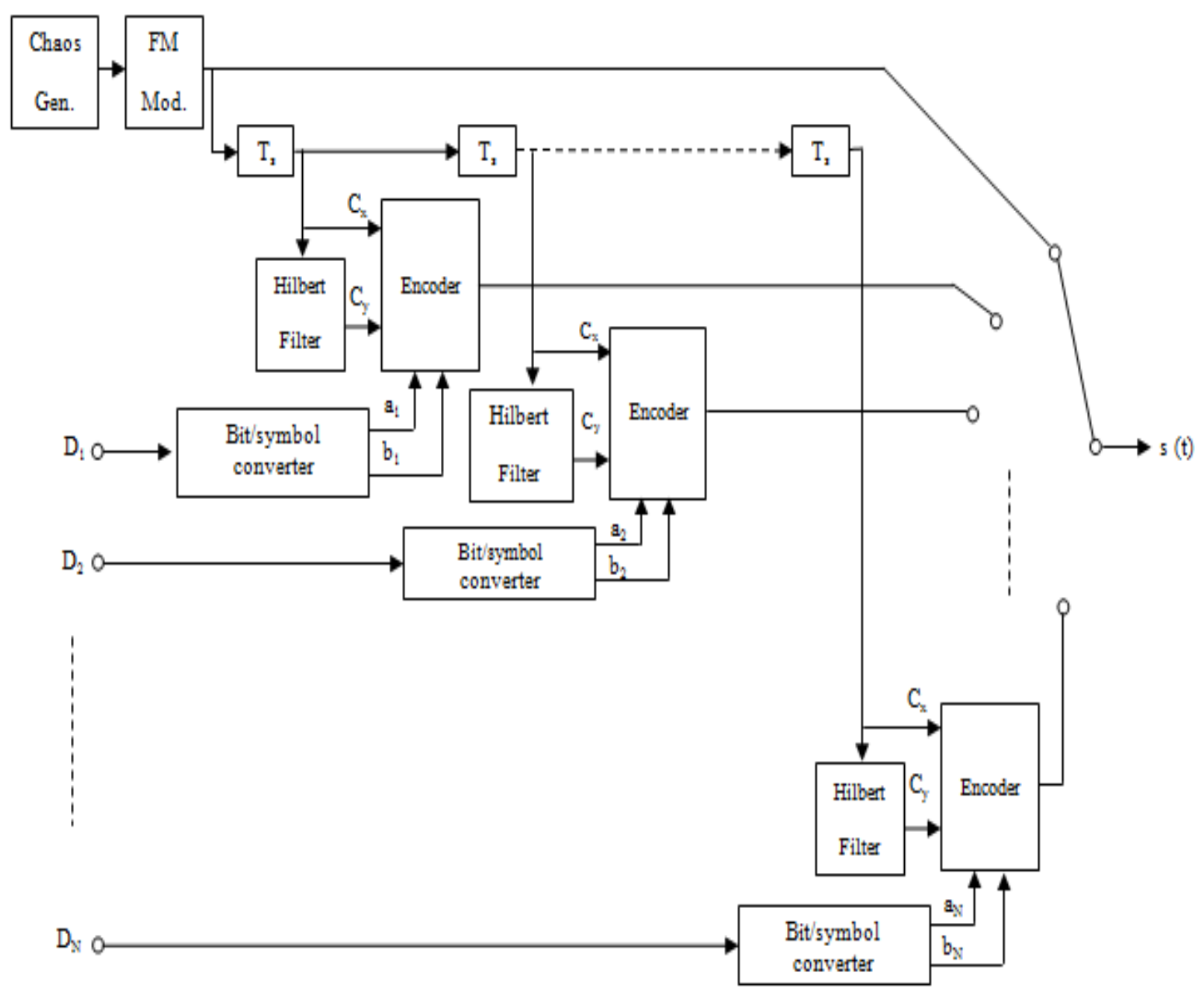

Figure 3. Enhanced FM-QCSK modulat or configuration implemented using a delay line having Ntaps 


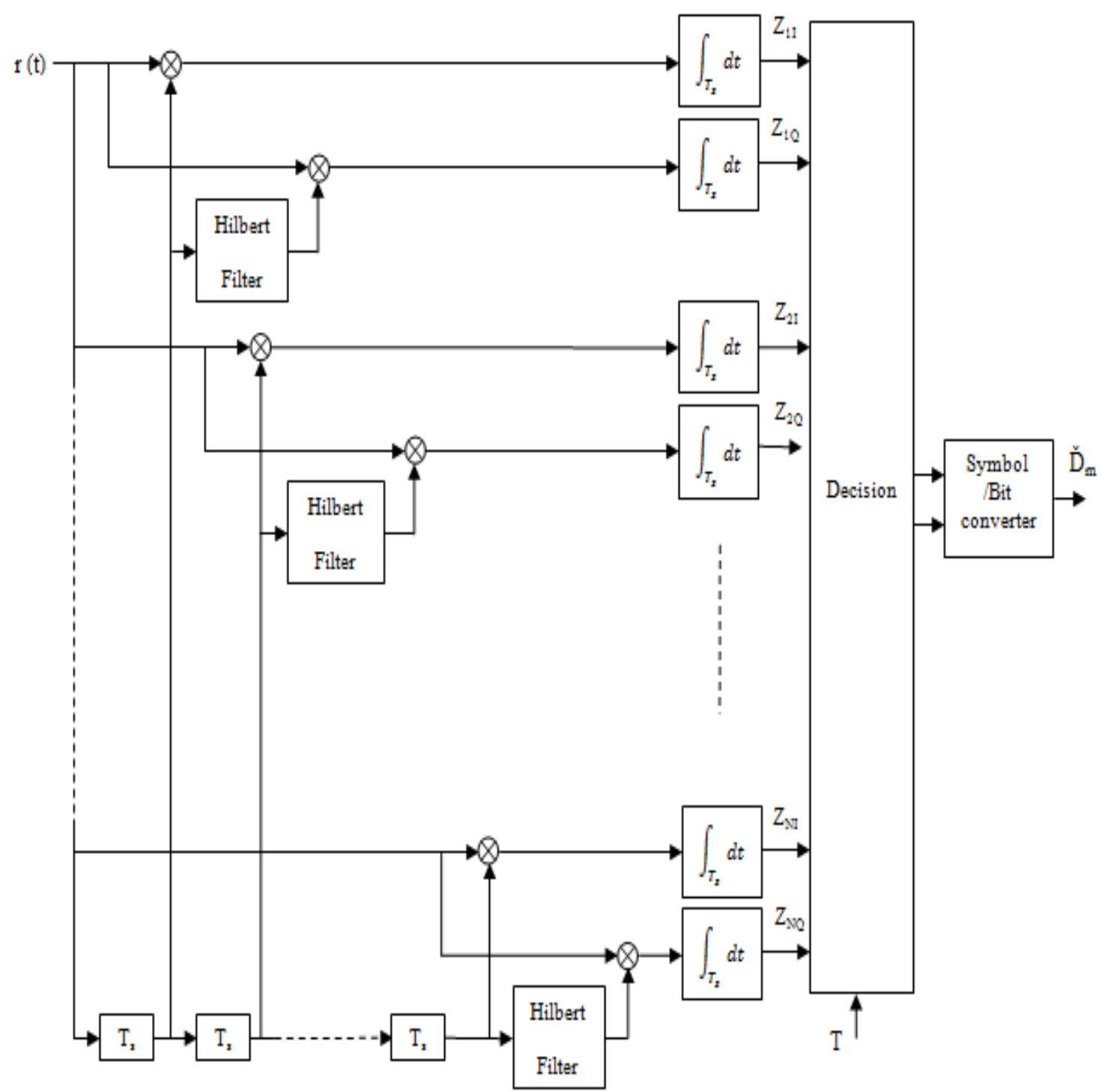

Figure 4. Enhanced FM-QCSK demodulat or configuration

Table 2. Elements of Observation Vectors at $\mathrm{T}_{\mathrm{s}}, 2 \mathrm{~T}_{\mathrm{s}}, \ldots(\mathrm{N}+1) \mathrm{T}_{\mathrm{s}}$ time instants

\begin{tabular}{|c|c|c|c|c|}
\hline Time instant & $\mathrm{z}_{1}$ & $\mathrm{z}_{2}$ & ....... & $\mathrm{z}_{\mathrm{N}}$ \\
\hline $\mathrm{T}_{\mathrm{s}}$ & - & - & ....... & - \\
\hline $2 \mathrm{~T}_{\mathrm{s}}$ & $\begin{array}{l}\breve{r}_{0}(t) * \breve{r}_{1_{I}}(t) \\
\breve{r}_{0}(t) * \breve{r}_{1_{Q}}(t)\end{array}$ & - & (n..... & - \\
\hline $3 \mathrm{~T}_{\mathrm{s}}$ & $\begin{array}{l}\breve{r}_{1}(t) * \breve{r}_{2_{I}}(t) \\
\breve{r}_{1}(t) * \breve{r}_{2_{I}}(t)\end{array}$ & $\begin{array}{c}\breve{r}_{0}(t) * \breve{r}_{2_{I}}(t) \\
\breve{r}_{0}(t) *{\breve{r_{2}}}_{1}(t)\end{array}$ & $\ldots \ldots \ldots$ & - \\
\hline$(\mathrm{N}+1) \mathrm{T}_{\mathrm{s}}$ & $\begin{array}{l}\breve{r}_{N-1}(t) * \breve{r}_{N_{I}}(t) \\
\breve{r}_{N-1}(t) * \breve{r}_{N_{I}}(t)\end{array}$ & $\begin{array}{l}\breve{r}_{N-2}(t) * \breve{r}_{N_{I}}(t) \\
\breve{r}_{N-2}(t) * \breve{r}_{N_{I}}(t)\end{array}$ & $\cdots \cdots \cdots$ & $\begin{array}{l}\breve{r}_{0}(t) * \breve{r}_{N_{I}}(t) \\
\breve{r}_{0}(t) * \breve{r}_{N_{I}}(t)\end{array}$ \\
\hline
\end{tabular}




\section{Performance Evaluation in AWGN Channel}

Based on the article by Yiwei Zhang, et al.[5], the BER of FM-QCSK is defined as follows:

$$
B E R_{F M-Q C S K}=\frac{1}{2} \operatorname{erfc}\left(\left(4 \frac{N_{0}}{E_{b}}+\frac{T}{2 T_{c}} \cdot \frac{N_{0}^{2}}{E_{b}^{2}}\right)^{-\frac{1}{2}}\right)
$$

where $T_{c}$ is the chip duration of discrete chaos signal. $\operatorname{In}[5]$, the term $\mathrm{T} /\left(2 \mathrm{~T}_{\mathrm{c}}\right)$ denoted as $\mathrm{K}$, which represent the number of chaotic samples in the reference signal. It is also shown in[5] that as K increase, the noise performance of QCSK is improved since it is a measure of the length of correlation interval. To get the BER of the enhanced FM-QCSK, we substitute the new values of bit duration $((\mathrm{N}+1) / \mathrm{N}) \mathrm{T}_{\mathrm{s}}$ and energy per bit $((\mathrm{N}+1) / \mathrm{N}) \mathrm{E}_{\mathrm{s}}$ in (9). In this way, the BER of enhanced FM-QCSK is:

$$
\begin{aligned}
& B E R_{\text {Enhanced FM-QCSK }}= \\
& \frac{1}{2} \operatorname{erfc}\left(\left(2 \frac{(N+1) N_{0}}{N E_{b}}+\frac{N T_{c}}{(N+1) T_{c}} \cdot \frac{(N+1)^{2} N_{0}^{2}}{4 N^{2} E_{b}^{2}}\right)^{-\frac{1}{2}}\right)
\end{aligned}
$$

\section{Simulation Results}

The chaotic spreading signal has generated by a discrete-time Hennon map: $x_{n+1}=1+g x_{n}+h x_{n}^{2}$ where $\mathrm{g}$ and $\mathrm{h}$ are constants with values $\mathrm{g}=-1.4$ and $\mathrm{h}=0.3$. The discrete signal is offset by -0.5 and scaled by 2 (to obtain zero mean) so that the signal range becomes[-1,1]. According to the article by Jiamin Pan and He Zhang[9], we define $\mathrm{T}_{\mathrm{c}}=0.05 \mu \mathrm{s}$ and $\mathrm{T}=4 \mu \mathrm{s}$. Subsequently, the FM modulator is defined as follows:

$$
\phi(t)=A_{c} \cos \left[\omega_{c} t+K_{f} \int_{-\infty}^{t} c(\alpha) d \alpha\right]
$$

In the above formula, $\mathrm{A}_{\mathrm{c}}=1 \mathrm{~V}, \mathrm{f}_{\mathrm{c}}=36 \mathrm{MHz}$, and $\mathrm{K}_{\mathrm{f}}=7.8$ $\mathrm{MHz} / \mathrm{V}$. Figure 5, shows the plot of BER versus $\mathrm{E}_{\mathrm{b}} / \mathrm{N}_{0}$ for conventional FM-QCSK $(\mathrm{N}=1)$ and the enhanced FM-QCSK for $\mathrm{N}=2,4,6,8$ and 10 in AW GN channel. The performance of BPSK (the best possible noise performance that can be achieved by any digital modulation scheme over AW GN) is also plotted for the purpose of performance comparison. It can be seen in this figure that by increasing $\mathrm{N}$, a significant improvement in the noise performance can be achieved. For example at $\mathrm{BER}=10^{-3}$, gains in $\mathrm{SNR}$ of $2 \mathrm{~dB}, 2.7 \mathrm{~dB}, 3.1 \mathrm{~dB}$, $3.3 \mathrm{~dB}$ and $3.4 \mathrm{~dB}$ when $\mathrm{N}=2,4,6,8$ and 10 respectively have been obtained using enhanced FM-QCSK scheme over the conventional one. Actually, the reason behind the improvement in the BER using the proposed scheme is that the noise associated with the received signal would be averaged due to the division of the correlation to $\mathrm{N}$ time slots. However, above certain limit increasing $\mathrm{N}$ has little effect on the system noise performance, i.e. threshold effect can be observed. We noticed in our simulations that this threshold occurs at $\mathrm{N}=8$. However, the performance can be also relatively improved by increasing $\mathrm{K}$ (the length of correlation interval).
Figure 6 shows the plot of BER versus $\mathrm{E}_{\mathrm{b}} / \mathrm{N}_{0}$ for conventional FM-QCSK $(\mathrm{N}=1)$ and the enhanced FM-QCSK for $\mathrm{N}=2,4,6,8$ and 10 in Rayleigh mult ipath fading channel. In this case we used in our simulations two paths; the second path delay was $75 \mathrm{~ns}$ with attenuation of $-3 \mathrm{~dB}$ which represents the specification of mult ipath environ ment inside office buildings. It is obvious in this figure that the performance of FM-QCSK is improved as $\mathrm{N}$ increased too. For example at $\mathrm{BER}=10^{-3}$, gains in SNR of $3 \mathrm{~dB}, 4.6 \mathrm{~dB}, 5.2 \mathrm{~dB}$, 5.6 and $5.8 \mathrm{~dB}$ when $\mathrm{N}=2,4,6,8$ and 10 respectively have been obtained. For $\mathrm{N}$ values greater than 8 , a saturation region is reached such that no more than $0.1 \mathrm{~dB}$ improvement can be gained. As compared with BPSK, enhanced FM-QCSK offered superior performance starting from $\mathrm{SNR}=5 \mathrm{~dB}$. For examp le at $\mathrm{BER}=10^{-3}$, mo re than $10 \mathrm{~dB}$ gain in SNR can be gained by using enhanced FM-QCSK with $\mathrm{N}=8$.

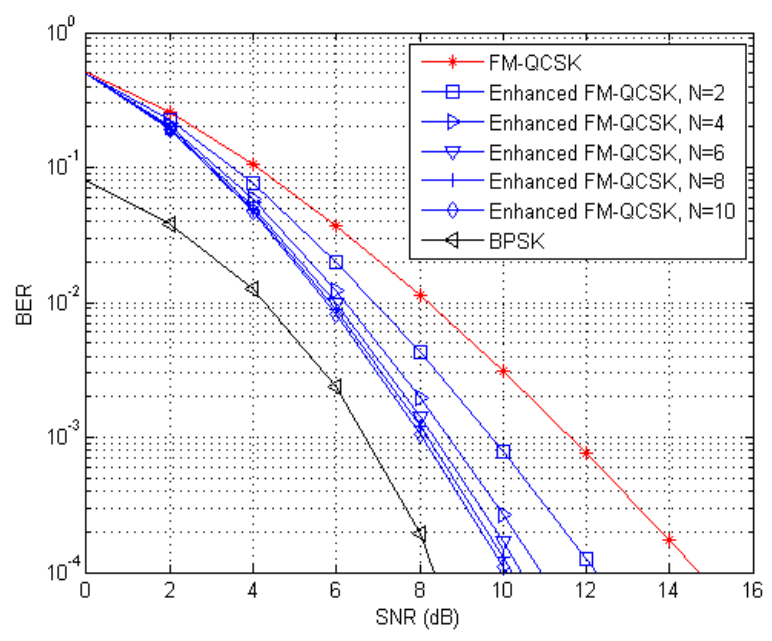

Figure 5. BER performance of conventional FM-QCSK, enhanced FM-QCSK for different values of N and BP SK over AWGN channel

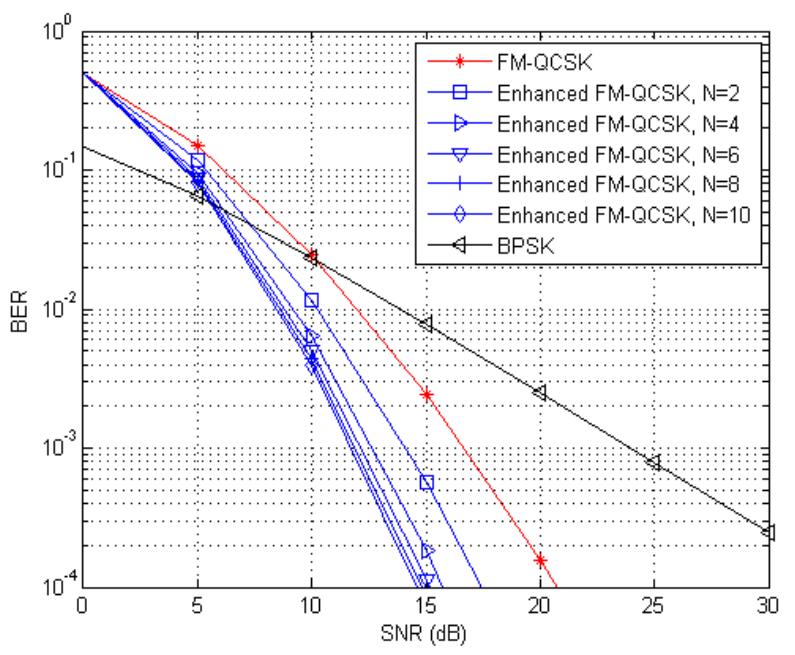

Figure 6. BER performance of conventional FM-QCSK, enhanced FM-QCSK for different values of $\mathrm{N}$ and BPSK over Rayleigh fading channel 


\section{Conclusions}

The increase of the data rate and the reduction in the energy consumption are important requirement for any modulation system. In FM-QCSK, these requirements can be optimized by making multi information-bearing chips associated with one reference chip. It has been shown by simulations in both AWGN and multipath fading channels that the performance of FM-QCSK system has been enhanced by introducing this optimization criteria. Furthermore, the bit error rate has been also reduced. For instant, $3 \mathrm{~dB}$ and $5 \mathrm{~dB}$ gains in SNR for AWGN and Rayleigh multipath fading channels respectively at $\mathrm{BER}=10^{-3}$ over the conventional one. The performance of the enhanced scheme is improved as the number of information bearing chips for one reference chip is increased up to a certain threshold $(\mathrm{N}=8)$ after which the system complexity is increased without gaining considerable improvement. A theoretical expression of the error probability for the enhanced scheme has been derived and its plot versus SNR is very closed to the simulation results. The possible future work can include analysing the effect of changing the correlation length on the system performance and hardware implementation of the proposed system.

\section{ACKNOWLEDGEMENTS}

The author would like to thank Prof. Dr. Alejandro Valenzuela from Bonn-Rhein-Sieg university of applied sciences in Germany for his scientific support and International Institute of Education IEE in USA for financing support of this research work.

\section{REFERENCES}

[1] G. Kolumban, et.al, "The role of synchronization in Digital Communications Using chaos-part II: Chaotic Modulation and Chaotic synchronization," IEEE Transactions on Circuits and Systems I, Vol.45, No.11, November 1998, pp. 1129-1140.

[2] M. P. Kennedy, et al, "Recent advances in aommunicating with chaos," IEEE International Symposium on Circuits and Systems (ISCAS'98), Vol4, Monterey 1998, pp.461-464.

[3] M.P. Kennedy, et al, "Performance evaluation of FM-DCSK modulation in multipath environment," IEEE Trans. Circuits and Systems I, Vol.47, No.12, December 2000, pp.1702-1710.

[4] Z. Galias, and G. M. Maggio, "Quadrature chaos-shift keying: theory and performance analysis," IEEE Transactions on Circuit and Systems. I, Vol.48, No.12, December 2001, pp.1510-1518.

[5] YiWei Zhang, et al, "Design and performance analysis of an FM-QCSK chaotic communication systems," International Conference on Wireless Communications, Networking and Mobile Computing (WiCOM), Wuhan 2006, pp.1-4.

[6] Z. Jàkó, "Performance improvement of DCSK modulation," Proceedings of International Workshop in Nonlinear Dynamics of Electronics Systems, (NDES'98), Budapest 1998, pp. 119-122.

[7] Chen Yiping, et al, "Performance of differential Chaos-Shift-Keying digital communication systems over several common channels," 2nd International Conference on Future Computer and Communication (ICFCC2010), Vol.2, Wuhan 2010, pp.755-759.

[8] Jinchao Tian and Xingzhau Zhang, "Design of Quadrature amplitude modulation based on chaos carrier," Journal of System Simulation, Vol.19, No.13, July 2007, pp.3123-3125.

[9] Jiamin Pan and He Zhang, "Design of FM-QACSK chaotic communication system,", International Conference on Wireless Communications and Signal Processing (WCSP2009), Nanjing 2009, pp.1184-1187.

[10] T. J. Wern and T. C. Yang, "Orthogonal Chaotic Vector shift keying in Digital Communications," IET Communications Journal, Vol.4, No.6, October 2009, pp. 739-753. 